\title{
Apoio social na autoestima e identidade social de pessoas trans brasileiras
}

\author{
Bruno de Brito Silva ${ }^{1}$ \\ Elder Cerqueira-Santos ${ }^{2}$ \\ ${ }^{1}$ Universidade Federal do Rio Grande do Sul, RS, Brasil \\ ${ }^{2}$ Universidade Federal de Sergipe, SE, Brasil
}

\begin{abstract}
Resumo
O objetivo do estudo foi investigar a influência e o papel da satisfação com o suporte social, do apoio familiar na identidade social e autoestima de travestis, homens e mulheres trans e pessoas não binárias. Fizeram parte do estudo 203 pessoas trans brasileiras(os), os quais responderam um questionário online com: escalas dos questionário Juventude Brasileira, escala de Necessidade de Identificação Social, escala de Autoestima de Rosenberg, e a de Satisfação com o Suporte Social. Análises de Variância (ANOVAs) e de Correlação de Pearson mostraram relações entre o apoio familiar e o escore de autoestima. Análises multivariadas de regressão linear mostraram que a idade, o fato de estar ou não em tratamento hormonal e a satisfação com o suporte social atuaram como variáveis associadas à identidade social dos participantes. Discute-se que apoio social pode atenuar o estresse psicológico da estigmatização, melhorar a autoestima e a identidade social trans, fornecendo suporte para violências perpetradas por uma sociedade heteronormativa.
\end{abstract}

Palavras-chave: Identidade de gênero; Transgênero; Identidade social; Autoestima; Apoio social.

\section{Social support on self-esteem and social identity of Brazilian transgender}

\begin{abstract}
The aim of this study was to explore to investigate the influence and role of satisfaction with social support, family support in social identity and self-esteem of traswomen, transmen and non-binary people. Were part 203 Brazilian transgender, who answered an online questionnaire with: scales of the questionnaires of Body Identity and of Brazilian Youth, Need for Identification Scale, Rosenberg Self-Esteem Scale and the Satisfaction with Social Support Scale. ANOVA and Pearson Correlations analysis showed relations between family support and the self-esteem score. Multivariate analysis of linear regression showed that age, the fact of whether or not undergoing hormone treatment and satisfaction with social support acted associated with the social identity of the participants. It is argued that social fostering can alleviate the psychological stress of stigmatization, improve self-esteem and to trans social identity providing support for violence perpetrated by a heteronormative society.
\end{abstract}

Keywords: Gender identity; Transgender; Social identity; self-esteem; Social support.

\section{Apoyo social en la autoestima y identidad social de personas transgénero brasileñas}

Resumen

El objetivo de este estudio fue explorar investigar la influencia y el papel de la satisfacción con el soporte social, del apoyo familiar en la identidad social y autoestima de travestis, hombres y mujeres trans, y personas no binarias. Participaron 203 personas trans brasileñas, quienes respondieron un cuestionario online con: las escalas del cuestionario Juventud Brasileña, la Escala de Necesidad de Identificación Social, Escala de Autoestima de Rosenberg, y la de Satisfacción con el soporte social. Análisis ANOVAs y la Correlación de Pearson mostraron relaciones entre el apoyo familiar y la puntuación de autoestima. Los análisis multivariados de regresión lineal mostraron que la edad, el hecho de estar o no en tratamiento hormonal y la satisfacción con el soporte social actuaron como variables asociadas a la identidad social de los participantes. Se discute que el apoyo social puede atenuar el estrés psicológico de la estigmatización, mejorar la autoestima y la identidade social trans, proporcionando apoyo a violencias perpetradas por una sociedad heteronormativa.

Palabras clave: Identidad de género; Transgénero; Identidad social; Autoestima; Apoyo social. 


\section{Introdução}

As travestilidades e as transexualidades são modos de existência que vão de encontro aos conceitos de masculinidade e feminilidade fortemente disseminados socialmente. Esses conceitos organizam as diversas práticas em sociedade, pois normatizam e exigem uma linearidade entre sexo designado ao nascimento, gênero, desejo e práticas sexuais (Bento \& Pelúcio, 2012). Travestis, homens e mulheres trans, e pessoas não binárias e integram o grupo social de pessoas transgênero que, para terem acesso às transformações corporais que reivindicam como condição para vivenciar a sua sexualidade, melhorar a autoestima e solidificar a identidade, podem ou não vir a se submeter a tratamentos hormonais e à cirurgias de afirmação de gênero (Silva \& Cerqueira-Santos, 2014).

Neste sentido, a identidade social se mostra um fenômeno de alta importância para qualquer indivíduo, pois estaria relacionada com o conhecimento da filiação a certos grupos sociais e com a significância emocional e valorativa que resulta desta filiação (Tajfel, 1972). E, de acordo com a Teoria da Identidade Social, o quanto o indivíduo se sente pertencente a determinado grupo influenciará a visão que o mesmo tem de si, dos outros e de como será cumprido seu papel social (Álvaro \& Garrido, 2007).

Além disso, é a partir desse processo de comparação entre grupos sociais, que se considera mais viável o surgimento de fenômenos como a formação de identidades, o que não significa que um indivíduo não possa ter uma identificação problemática com seus grupos de pertença (Álvaro \& Garrido, 2007; Sousa \& Suda, 2006; Tajfel, 1981). No caso da identidade trans, a mesma poderia ser encarada enquanto uma categoria organizadora da experiência e da trajetória individual. Porém, poderia também ser considerada como um categoria identitária temporária, utilizada apenas para o acesso a instituições que assistem demandas como tratamento hormonal e cirurgias da afirmação de gênero, e que num momento posterior a este atendimento, a pessoas trans buscassem eliminar este rótulo e se identificar enquanto pessoas binário, homem ou mulher, ou enquanto pessoa não binário, de um gênero "fluido" (Almeida, 2012). Desse modo, não haveria uma identidade transexual ou travesti em si, mas sim "posições de identidade" que se dariam por meio da aceitação ou negação dos modelos de masculinidade e feminilidade socialmente disponibilizados (Bento, 2006).

A teoria da identidade social afirma que um indivíduo somente preservará as contribuições de um grupo em sua identidade social se o mesmo trouxer avaliações positivas à sua autoimagem (Tajfel, 1981; Deschamps, 2009). Ao se criar, realizar ou alcançar algum objetivo por si mesma, a pessoa se sentirá bem sobre si e sobre suas habilidades, e alcançará essa autoimagem positiva, sentindo-se satisfeita com as características que reconhece em si mesmo (Nelson, 2002). E é essa alta autoestima pessoal, considerada na teoria da identidade social como autoimagem positiva, que traz uma sensação de bem estar e prazer (Barišic, Milosavljevic, Duišin, Batinic, Vujovic, \& Milovanovic, 2014).

A autoestima pessoal está relacionada a como uma pessoa se sente sobre suas capacidades, habilidades, aptidões e seu modo de ser de maneira geral, e acarreta uma atitude positiva ou negativa do indivíduo em direção a si mesmo enquanto totalidade (Rosenberg, Schooler, Schoenbach, \& Rosenberg, 1995). Todavia, a capacidade limitada ou deficiente de se enxergar de maneira favorável e completa pode tornar as pessoas susceptíveis a dificuldades de adaptação às situações adversas, como ao preconceito e a discriminação (Barišic et al., 2014).

Neste sentido, quando essa autoimagem positiva não é alcançada pelos feitos pessoais, será obtida ou restabelecida ao se considerar a criação, realização e alcance de objetivos por meio dos grupos que pertence (Nelson, 2002). É essa necessidade da autoimagem positiva, que abastece motivação e cognição em prol do bem estar, que faz com que pessoas que integram grupos historicamente marginalizados, repartam sentimentos e estabeleçam uma conexão com outros indivíduos que são estigmatizados pelas mesmas características (Nelson, 2002; Sánchez \& Vilain, 2009).

Com relação a este aspecto, uma estratégia de enfretamento capaz de amenizar os efeitos da opressão exercida pela sociedade é nutrir uma identificação positiva, e uma avaliação desta identificação, a um determinado grupo social, conhecida também como autoestima coletiva (Crocker \& Luhtanen, 1990; Sánchez \& Vilain, 2009). Porém, pode-se argumentar que o sofrimento psicológico derivado do medo da discriminação e pelo simples fato de ser membro de grupos sociais pouco ou nada valorizados acaba por causar uma baixa autoestima coletiva e também uma baixa autoestima global entre os indivíduos trans (Bockting, Benner, \& Coleman, 2009; Crocker \& Luhtanen, 1990; Sánchez \& Vilain, 2009).

Além disso, a falta de suporte social por parte da maioria dos grupos aos quais as pessoas trans integram (família, pessoas do trabalho, escola ou universidade/faculdade, comunidade do bairro residente, profissionais de atendimento à saúde LGBT) é uma realidade para a maioria destes sujeitos e acaba 
sendo necessário trabalhar com a ideia de uma rede social enfraquecida (Silva \& Cerqueira-Santos, 2014). Neste sentido, são importantes o estabelecimento e a consolidação de relações que carregam em si o caráter de apoio, gerando a interação de grupos que formam uma rede (Costa \& Dell'Aglio, 2009; Paludo \& Koller, 2005).

O apoio social pode ser conceituado, portanto, como o conjunto de relações próximas e significativas que representam o suporte recebido e percebido pelos indivíduos provenientes de recursos pessoais, profissionais e institucionais ao longo da vida, independente da constância destes recursos com a finalidade de oferecer assistência aos mesmos, principalmente em situações de risco (Costa \& Dell’Aglio, 2009; Paludo \& Koller, 2005).

Dentre as principais fontes de apoio social das pessoas trans estariam: as amizades, por meio do suporte emocional, afetivo, informativo e de interações positivas proveniente dos pares e na comunidade, além da família, por meio da existência de coesão, amor parental, consistência disciplinar, relação conjugal positiva e ser proprietário de uma moradia (Costa \& Dell'Aglio, 2009; Paludo \& Koller, 2004; Soares, Feijó, Valério, Siquieri, \& Pinto, 2011; Winck \& Petersen, 2005). Dessa forma, o apoio familiar, que é entendida aqui como a forma de suporte social proveniente da família de origem, por meio da aceitação e coesão da família pode atuar como preditora de maior autoestima, maior apoio social e estado geral de saúde, além de proteger contra a depressão, abuso de substâncias, ideação e comportamentos suicidas (Ryan et al., 2010). Em adição, este tipo de apoio está relacionado idade mais tardia da primeira relação sexual, eficácia sexual, um emprego mais estável, além da melhoria da qualidade dos relacionamentos familiares e da satisfação com a vida (Grossman, D’Augelli \& Frank, 2011; Stotzer, 2011).

Ademais, outro elemento constituinte da rede de apoio social trans é a comunidade por meio da disponibilidade de serviços públicos e sociais, como as Organizações não Governamentais voltadas para a população LGBT, de qualidade e a baixa incidência de violência nos locais aos quais o indivíduo frequenta (Bento, 2006; Bento, 2009; Soares et al., 2011; Winck $\&$ Petersen, 2005).

Dessa forma, pressupõe-se que a construção da identidade social e a autoestima da pessoa trans no contexto atual é fortemente influenciada e determinada pela relação com as redes de apoio sociais estabelecidas. Nesse sentido, o presente artigo tem por objetivo investigar a influência e o papel da satisfação com o suporte social, do apoio familiar na identidade social e autoestima de travestis, homens e mulheres trans e pessoas não binárias. Busca-se também avaliar a identidade social e a autoestima, bem como o suporte social recebido destas pessoas.

\section{Método}

\section{Delineamento}

Este estudo tem delineamento descritivo e explicativo de corte transversal e caráter analítico quantitativo, tendo abordagem online (survey), através do levantamento acerca das variáveis identidade social, autoestima e apoio social de travestis, homens e mulheres trans e pessoas não binárias.

\section{Participantes}

A amostra teve caráter não probabilístico por conveniência, vide locais mencionados nos procedimentos para coleta de dados e foi formada por brasileiras(os) que se identificaram enquanto travestis, mulheres, homens trans ou pessoas não-binárias, maiores de 18 anos. Foram retirados da amostra questionários que apresentavam mais da metade das questões não respondidas $(n=38)$, bem como sujeitos não brasileiros $(n=4)$. Assim, o presente estudo foi formado por 203 participantes.

\section{Instrumentos e Procedimentos}

O instrumento se constituiu por um questionário autoaplicável, exposto por meio do software e ferramenta de questionários online Survey Monkey e pela utilização de mensagens, e-mails, e mídias sociais para a divulgação da pesquisa.

$\mathrm{O}$ instrumento da presente pesquisa foi composto por 28 questões e dividido em seis partes. Para este estudo foram analisadas as seguintes questões:

Dados sociobiodemográficos. Estes dados são referentes à caracterização dos aspectos mais básicos do grupo estudado como cidade (região do País), cor de pele, idade, profissão, escolaridade, sexo designado ao nascimento, identidade de gênero, orientação sexual, crença religiosa, renda, presença de família e filhos, e moradia com companheiros (as).

Escalas do Questionário Juventude Brasileira. O Questionário da Juventude Brasileira foi elaborado para a segunda etapa do Estudo Nacional sobre Fatores de Risco e Proteção na Juventude Brasileira (Koller, Cerqueira-Santos, Morais, \& Ribeiro, 2005). Para o presente estudo, foram utilizadas duas escalas do questionário supracitado:

\footnotetext{
1. Escala do tipo Likert Nível de Apoio Social Familiar - formada pelos pontos "Discordo", "Nem concordo, nem discordo" e "Concordo",
} 
com itens que correspondiam à avaliação do apoio proveniente do grupo familiar. Esta última escala foi composta por nove itens, e apresenta consistência interna Alpha de Cronbach de 0,80 (Costa \& Dell'Aglio, 2009).

2. Escala padronizada constituída pelos itens da Escala de Autoestima de Rosenberg (1989), adaptada por Hutz (2000) para o Brasil possui dez itens, sendo seis referentes a uma visão positiva de si mesmo e quatro referentes a uma visão autodepreciativa. As opções de resposta são "Nunca", "Quase nunca", "Às vezes", "Quase sempre", "Sempre" e o Alpha de Cronbach da escala referida é de 0,70.

Escala Necessidade de Identificação Social (nID). Esta escala procura investigar as diferenças individuais na propensão para se identificar com grupos sociais, por meio das crenças sobre um grupo se tornar autorreferencial e autodefinidor das características dos sujeitos que o integram. É uma escala Likert composta pelos pontos "Concordo totalmente", "Concordo na maior parte", "Nem concordo, nem discordo", "Discordo na maior parte", "Discordo totalmente", sendo o Alpha de Cronbach de 0,89, para a presente amostra, e uma estrutura bifatorial de seis itens que avaliam a autodefinição do sujeito e de cinco itens que acessam questões relativas ao pertencimento em grupos (Mayhew, Gardner, \& Achkanasy, 2010). Para a apresentação desta escala, solicitava-se que os participantes respondessem a mesma pensando em seu(s) grupo(s) de identidade de gênero e sexual de pertença.

Escala de Satisfação com o Suporte Social (ESSS). A ESSS foi construída para medir a satisfação com o suporte social existente, afirmando que as medidas de percepção do suporte social que se recebe explicam melhor a saúde do que as de suporte social por fatores mais externos. A consistência interna da escala (Alpha de Cronbach) é de 0,86. Além disso, a escala é composta por 15 itens com alternativas de resposta variando entre "Concordo totalmente", "Concordo na maior parte", "Nem concordo, nem discordo", "Discordo na maior parte", "Discordo totalmente", contendo três fatores que investigam a satisfação com amizades $(\alpha=0,83)$, intimidade $(\alpha=0,74)$ e satisfação com a família $(\alpha=0,74)$ (Paris-Ribeiro, 1999).

\section{Procedimentos de Coleta de Dados}

O link foi enviado por e-mails, listas de ONGS, gerando o repasse para o público alvo da presente pesquisa. Além da divulgação por meio da página da mídia social facebook "De todas as cores", que falava sobre temáticas relacionadas ao público LGBT. Esta divulgação foi feita através da inserção em grupos de caráter privado ou não, que fossem direcionados a travestis, homens e mulheres trans, crossdressers, pessoas transgênero que buscavam discutir desde questões relativas a gênero e sexualidade até proporcionar uma rede de apoio social.

\section{Aspectos Éticos da Pesquisa}

O Termo de Consentimento Livre e Esclarecido (TCLE) foi apresentado em uma página inicial e o ato de clicar no botão "Aceito", para continuação do questionário, significava a concordância com a participação, vide anexo I.

Além disso, foram salvaguardadas as diretrizes éticas pré-estabelecidas para a prática da pesquisa científica envolvendo seres humanos, conforme a resolução 466/12 do CNS (Brasil, Ministério da Saúde, 2012). O estudo foi aprovado pelo Comitê de Ética em Pesquisa com Seres Humanos da Universidade Federal de Sergipe, sob o protocolo 09234912.4.0000.0058.

\section{Tratamento e Análise dos dados}

Foi construído um banco de dados anexo e utilizouse o SPSS v20. Inicialmente, foram feitas análises estatísticas exploratórias e descritivas para determinar a frequência (percentual e absoluta) e a amplitude de todas as variáveis do estudo. Em seguida, as relações entre todos os participantes e os grupos de análise de identidade de gênero masculina e feminina foram computadas, segundo análises descritivas paramétricas e de variância (Testes $t$ e ANOVA oneway). A ANOVA foi utilizada para avaliar a existência de diferenças entre as médias dos grupos. Após, utilizou-se a Correlação de Pearson para verificar quais variáveis influenciariam na identidade social, na autoestima, no apoio familiar e no suporte social com um todo. E, por fim, foram realizadas Regressões Lineares para estimar quais variáveis influiriam na autoestima e na identidade social a partir do grupo de homens trans, travestis e mulheres trans. $\mathrm{O}$ nível de significância utilizado foi de $p<0,05$.

\section{Resultados}

Inicialmente, serão apresentados resultados relativos aos dados sociobiodemográficos dos(as) participantes. Em seguida, serão apresentadas as análises bivariadas (Teste $t$ e ANOVAs e Correlação de Pearson) e multivariadas (Regressões Lineares) entre as variáveis de estudo por meio dos escores gerados a partir das perguntas e escalas do instrumento.

\section{Dados sociobiodemográficos}


Dentre os 203 respondentes da pesquisa, a média de idade foi de 27,35 anos, $\mathrm{DP}=8,71 \quad(n=203)$, com mínimo de 18 e o máximo de 63 anos de idade. Além disso, no que diz respeito aos dados amostrais com relação ao sexo designado ao nascimento, $55,2 \%$ $(n=112)$ declararam nascer com o aparato biológico e fisiológico masculino.

No que diz respeito à cor, $54,7 \%(n=111)$ se autodenominou enquanto "branco" e em relação à crença, a maioria disse não ter religião, mas acreditar em Deus, com 38,4\% $(n=78)$ das repostas. Além disso, a escolaridade foi uma variável em que $31 \%$ $(n=63)$ dos participantes apontaram ter grau superior completo, seguido de $27,6 \%$ que relataram ter grau médio completo $(n=56)$. A renda média apontada dos sujeitos do estudo foi de $\mathrm{R} \$ 3919,07$ ( $\mathrm{DP}=7264,11)$.

No que concerne ao estado civil ou cohabitacional, $45,3 \%(n=92)$ dos respondentes são solteiros e não possuem namorado(a) ou companheiro(a), seguidos de $29,6 \%(n=60)$ que relataram ser solteiro(a)s, mas possuírem namorado(a) ou companheiro(a). Em outro momento, quando questionados acerca do fato de possuírem namorado(a), companheiro(a) ou amante, $29,6 \%(n=60)$ dos(as) participantes da pesquisa responderam de forma afirmativa. E, no que se refere a possuírem filhos, responderam que "sim", apenas 5,9\% $(n=12)$ da amostra.

Além disso, foi feita uma categorização com relação as variáveis cidade ou estado indicado como de origem distribuindo as repostas nas cinco regiões do Brasil, e percebeu-se uma maior incidência de indivíduos da região sudeste $(n=85)$, seguido da região nordeste $(n=45)$, região sul $(n=29)$, centro-oeste $(n=23)$ e região norte $(n=21)$.

Quanto ao fato de se fazer tratamento hormonal para a transexualização, 46,3\% $(n=94)$ alegaram já haver passado ou estarem passando pelo tratamento referido, e, 96,1\% $(n=195)$ dos sujeitos da presente pesquisa, relataram não ter realizado a cirurgia de redesignação genital ou de afirmação de gênero.

Com relação à identidade de gênero dos participantes, também foram realizadas categorizações, e a mesma variável se subdividiu em três categorias:

- "Homens trans - Com 35,5\% $(n=72)$ das respostas, que seriam referentes à participantes que relataram ter o sexo designado ao nascimento masculino e pertencer a este gênero ou serem homens transexuais que se sentiam enquanto predominantemente pertencentes ao gênero masculino.

- "Não binária" - Nesta categoria, que obteve percentual de $24,9 \%(n=50)$, foram incluídas todas as respostas que iam de encontro ao paradigma binário da sexualidade (classificação do gênero em duas formas distintas, opostas e desconectadas, que seriam o feminino e o masculino) como a exemplo das pessoas que se consideraram pertencentes a um "gênero fluido", pertencentes ao "gênero transexual", ou ao "gênero travesti", "bigênero", "agênero", dentre tantas outras assertivas que reafirmavam a problematização do gênero.

- "Travestis e mulheres trans" - Incluiu todas as respostas relacionadas a indivíduos que alegaram possuir a genitália masculina, mas se sentirem enquanto mulheres, bem como mulheres transexuais que relataram como se percebendo enquanto integrantes do gênero feminino, categoria esta que totalizou $34,8 \%$ $(n=70)$ das repostas.

Dentre essas três categorias, houveram nove sujeitos $(4,4 \%$ dos respondentes) que não souberam definir ou aderiram respostas que não se enquadravam nas categorias.

\section{Análises Bivariadas das variáveis principais com os dados sociobiodemográficos}

Em relação à variável idade foi realizada uma categorização em dois grupos por meio da média de idade dos participantes em mais novos (abaixo de 27,35 anos) e mais velhos (acima de 27,35 anos). Com isso, realizou-se testes $t$ para a idade e foram encontradas diferenças estatisticamente significativas com relação à autoestima $[t(182)=-2,09 ; p<0,02]$, onde os mais velhos tiveram maiores pontuações na escala, mas não foi encontrada relação estatisticamente significativa para com a identidade social, apoio familiar e suporte social.

As ANOVAs realizadas entre os tipos de identidade de gênero e os índices de identidade social, autoestima e apoio familiar não indicaram diferenças estatisticamente significativas entre os grupos $(p>0,05)$. Foram constatadas diferenças estatisticamente significativas apenas para o escore de suporte social $[F(3,137)=3,407 ; p<0,02]$, e por meio do teste post $h o c$ de Tukey, percebeu-se que as diferenças se davam entre os grupos de Homens trans ou aqueles que se identificaram com o gênero masculino que tiveram uma maior pontuação do que os que não souberam definir sua identidade de gênero $(p<0,05)$.

NaANOVAs com relação aos níveis de escolaridade, foi evidenciado diferenças significativas para o índice de identidade social $[F(3,145)=5,120 ; p<0,003]$, e ao se realizar o teste post hoc de Tukey, percebeu-se que o grupo que tinha apenas cursado até o ensino médio diferiu obtendo menores médias do que os(as) que estavam na pós-graduação $(p<0,002)$. Não foram encontradas diferenças estatisticamente significa- 
tivas em relação a escolaridade para com as outras variáveis.

No que concerne ao estado civil, as ANOVAs apontaram que os grupos diferiram de maneira significativa para os índices de apoio familiar $[F(2,176)=3,469 ; p<0,04]$. Pelas análises do teste post hoc de Tukey, constatou-se diferenças entres solteiros(as) sem namorado(a) e os que vivem com companheiro(a) ou casados(as) $(p<0,04)$, que obtiveram maiores médias no índice de apoio familiar.

Já em relação ao teste $t$ realizado para o fato de ter ou não passado pela cirurgia de redesignação genital (ou de afirmação de gênero), observou-se diferenças estatisticamente significativas para com o índice de apoio familiar $[t(182)=-2,06 ; p<0,05]$, no qual as médias daqueles que já haviam passado por tal procedimento foram maiores do que aqueles que não passaram.

\section{Análises Bivariadas entre Autoestima, Identidade social e suporte social}

Foram realizadas divisões pelo percentil 33 e posterior categorização em três níveis os índices das variáveis autoestima e a identidade social. Em seguida, ao serem conduzidas as ANOVAs entre os escores de autoestima, apoio familiar, suporte social e os três níveis de identificação aos grupos de pertença, percebeu-se diferenças estatisticamente significativas apenas para a variável suporte social $[F(2,130)=3,20 ; p<0,05]$, e não para as outras variáveis. De acordo com o teste post hoc de Tukey utilizado, percebeu-se diferenças entre aqueles pouco ou nada identificados aos grupos de pertença e aqueles identificados ou muito identificados ao grupo de pertença, obtendo estes maiores médias na escala de suporte social $(p<0,04)$.

Já ao serem realizadas as ANOVAs entre identidade social, apoio familiar, suporte social e os três níveis de autoestima, observou-se diferenças estatisticamente significativas apenas para a variável apoio familiar $[F(2,178)=6,94 ; p<0,002]$, e não para as outras variáveis. Ao se aplicar o teste post hoc de Tukey, percebeu-se diferenças entre aqueles com nível baixo e os com nível médio de autoestima, os quais apresentaram maiores médias de apoio familiar $(p<0,002)$.

TABELA 1

ANOVAs entre autoestima, apoio familiar, suporte social e os três níveis de identificação aos grupos de pertença

\begin{tabular}{|c|c|c|c|c|c|c|c|c|c|}
\hline \multirow[b]{2}{*}{ Niveis de Identidade Social } & \multicolumn{3}{|c|}{ Autoestima } & \multicolumn{3}{|c|}{ Suporte Social } & \multicolumn{3}{|c|}{ Apoio Familiar } \\
\hline & $\begin{array}{c}M \\
(D P)\end{array}$ & $t / F$ & $P$ & $\begin{array}{c}M \\
(D P)\end{array}$ & $t / F$ & $P$ & $M(D P)$ & $t / F$ & $P$ \\
\hline Pouco ou nada identificado & $\begin{array}{l}32,98 \\
(3,12)\end{array}$ & 0,37 & 0,69 & $\begin{array}{l}38,71 \\
(8,57)\end{array}$ & 3,20 & 0,044 & $\begin{array}{l}19,91 \\
(5,83)\end{array}$ & 0,32 & 0,72 \\
\hline Nem muito nem pouco & $\begin{array}{l}32,50 \\
(3,49)\end{array}$ & & & $\begin{array}{l}41,48 \\
(5,57)\end{array}$ & & & $\begin{array}{l}20,50 \\
(5,46)\end{array}$ & & \\
\hline Identificado ou muito & $\begin{array}{l}32,50 \\
(3,23)\end{array}$ & & & $\begin{array}{l}42,42 \\
(7,73)\end{array}$ & & & $\begin{array}{l}19,53 \\
(5,24)\end{array}$ & & \\
\hline
\end{tabular}

TABELA 2

ANOVAs entre identidade social, apoio familiar, suporte social e os três níveis de autoestima

\begin{tabular}{|c|c|c|c|c|c|c|c|c|c|}
\hline \multirow[b]{2}{*}{ Níveis de Autoestima } & \multicolumn{3}{|c|}{ Identidade Social } & \multicolumn{3}{|c|}{ Suporte Social } & \multicolumn{3}{|c|}{ Apoio Familiar } \\
\hline & $\begin{array}{c}M \\
(D P)\end{array}$ & $t / F$ & $P$ & $\begin{array}{c}M \\
(D P)\end{array}$ & $t / F$ & $P$ & $\begin{array}{c}M \\
(D P)\end{array}$ & $t / F$ & $P$ \\
\hline Baixo & $\begin{array}{l}28,66 \\
(8,21)\end{array}$ & 1,97 & 0,14 & $\begin{array}{l}41,37 \\
(6,89)\end{array}$ & 0,97 & 0,38 & $\begin{array}{c}18,59 \\
(5,05)\end{array}$ & 6,94 & 0,001 \\
\hline Médio & $\begin{array}{l}27,90 \\
(9,74)\end{array}$ & & & $\begin{array}{l}39,18 \\
(7,42)\end{array}$ & & & $\begin{array}{l}21,95 \\
(5,44)\end{array}$ & & \\
\hline Alto & $\begin{array}{l}31,85 \\
(10,3)\end{array}$ & & & $\begin{array}{l}40,57 \\
(10,0)\end{array}$ & & & $\begin{array}{l}19,88 \\
(5,40)\end{array}$ & & \\
\hline
\end{tabular}


Análises de correlação de Pearson foram realizadas com base nos resultados significativos apresentados acima e em modelo teórico de hipótese de relação entre as variáveis. Para a variável índice de identidade social foi encontrada uma correlação positiva significativa o índice de suporte social $(p<0,001)$.

No que diz respeito ao índice de autoestima, verificou-se correlações positivas significativas com o apoio familiar $(p<0,02)$, entre o índice de suporte social e os de apoio familiar $(p<0,03)$, e entre idade e apoio familiar $(p<0,002)$.

TABELA 3

Correlação de Pearson entre identidade social, apoio familiar, suporte social e os três níveis de autoestima

\begin{tabular}{|c|c|c|c|c|c|}
\hline & & 2 & 3 & 4 & 5 \\
\hline \multirow[t]{3}{*}{1} & $R$ & 0,007 & 0,125 & $-0,032$ & 0,242 \\
\hline & $P$ & 0,928 & 0,092 & 0,708 & 0,001 \\
\hline & $N$ & 150 & 184 & 142 & 184 \\
\hline \multirow[t]{3}{*}{2} & $R$ & & 0,047 & 0,331 & 0,059 \\
\hline & $P$ & & 0,575 & $\mathbf{0 , 0 0 0}$ & 0,479 \\
\hline & $N$ & & 146 & 133 & 148 \\
\hline \multirow[t]{3}{*}{3} & $R$ & & & 0,087 & 0,189 \\
\hline & $P$ & & & 0,309 & 0,011 \\
\hline & $N$ & & & 140 & 181 \\
\hline \multirow[t]{3}{*}{4} & $R$ & & & & $-0,191$ \\
\hline & $P$ & & & & 0,023 \\
\hline & $N$ & & & & 141 \\
\hline
\end{tabular}

1 - Idade; 2 - Índice de Identidade Social; 3 - Índice de Autoestima; 4 -Indice de Suporte social; 5 -Índice de apoio familiar

\section{Análises multivariadas entre identidade social e autoestima e as variáveis de apoio social}

Foram realizadas análises multivariadas tendo como variável desfecho os escores encontrados a partir das escalas de necessidade de identificação social e da escala de Autoestima de Rosenberg. O modelo final apresentou variância explicada de 38,9\% para identidade social e de $16,5 \%$ para a autoestima, que considera como variáveis preditoras a idade, tratamento hormonal, autoestima ou identidade social, a depender da variável de desfecho, suporte social, apoio familiar.

A idade, o fato de usar ou fazer tratamento com hormônios, e o escores da ESSS (Escala de Satisfação com o Suporte Social) são as variáveis mais significativas para associação com o desfecho identidade social. Já em relação ao desfecho da autoestima, a variáveis utilizadas não apresentaram significância estatística.
TABELA 4

Regressão linear para o escore em relação à identidade social

\begin{tabular}{lcccc}
\hline Modelo: Identidade Social & Carga & $\begin{array}{c}\text { Erro } \\
\text { Padrão }\end{array}$ & Beta & $P$ \\
Idade & 0,042 & 0,016 & 0,415 & $\mathbf{0 , 0 1 2}$ \\
Tratamento Hormonal & $-0,621$ & 0,262 & $-0,372$ & $\mathbf{0 , 0 2 3}$ \\
Autoestima & 0,008 & 0,034 & 0,032 & 0,820 \\
Suporte Social & 0,041 & 0,016 & 0,341 & $\mathbf{0 , 0 1 5}$ \\
Apoio Familiar & 0,028 & 0,027 & 0,180 & 0,321 \\
\hline
\end{tabular}

\section{Discussão}

O objetivo do presente artigo foi investigar investigar a influência e o papel da satisfação com o suporte social, do apoio familiar na identidade social e autoestima de travestis, homens e mulheres trans e pessoas não binárias. No que concerne aos dados desta pesquisa é importante observar que, a amostra foi constituída por um número proporcional de homens trans $(n=72)$ e de travestis e mulheres trans $(n=70)$. Porém, o que é interessante observar é a alta porcentagem de sujeitos que se consideraram pertencentes a um gênero "não-binário" (24,9\%). Diante disso, o público participante da pesquisa coloca a problemática da multiplicidade do fazer gênero em questão, na qual há novas possibilidades para além do que seria considerado inteligível culturalmente pelos discursos médicos, jurídicos e até mesmo socioculturais, que imprimem os chamados "gêneros inteligíveis", presos a coerência binária, e a heteronormatividade que rege as práticas em sociedade (Próchno \& Rocha, 2011).

No que se refere à identidade social, todas as análises realizadas (ANOVAs, correlações de Pearson e a Regressão linear) apontam a influência da satisfação com o suporte social para esta variável. Concernente a este aspecto, a própria teoria da identidade social mostra que é na base da avaliação de si mesmo que se encontra a identidade social, e que esta, estaria "ligada ao conhecimento e avaliativo que resulta da pertença a determinados grupos sociais" (Deschamps, 2009, p. 63). De acordo com Costa e Dell'Aglio (2009), as relações que se originam da rede de apoio social são capazes não só de oferecer apoio e influenciar o desenvolvimento do comportamento de suporte, mas também aumentar recursos do indivíduo, condicionando o grau de apego, integração social, autovalorização e orientação.

Outra característica importante na identidade social trans é a identidade de gênero que também mostrou relação com a satisfação com o suporte social mostrando que Homens trans ou aqueles que se identificaram com 
o gênero masculino tiveram uma maior pontuação do que aqueles que não souberam definir sua identidade de gênero. No que engloba esse aspecto da importância da identidade de gênero para a identidade social e para o suporte social, pode-se afirmar que é na pertença aos grupos sociais que os indivíduos enfrentam o desejo de se sentirem relativamente semelhantes ao outro, mas conservando sempre suas características individuais (Deschamps, 2009).

Em adição, observou-se, no presente estudo, que a identidade social da mulher trans, do homem trans e da travesti está em constante contato e recebe influência contínua do meio social no qual estes sujeitos se inserem. Dessa forma, como o indivíduo se avalia e como percebe os grupos de pertença (identidade social) tem estreita conexão com como a família, os pares, e os cônjuges/namorados, fornecem apoio e suporte a essas "outras" formas de se sentir mulher/homem ou de vivenciar o gênero (Bento, 2009).

Outras variáveis que apresentaram influência sobre a identidade social foram a escolaridade e a idade. $\mathrm{O}$ fato do indivíduo com maior escolaridade ter apresentado maiores médias na ANOVA com o escore da escala de necessidade de identificação social pode ter se dado porque, em geral, um maior nível de instrução dá oportunidade de acesso a mais informações sobre formas de preconceito e discriminação e de como lidar com a mesma se empoderando de seu modo de existência trans (Bento, 2006). Ao se considerar essa assertiva, de acordo com o relatório de 2011 do National Transgender Discrimination Survey (NTDS), survey realizado nos EUA sobre discriminação com a população trans, mostra que este grupo frequentemente não consegue ter acesso a oportunidades educacionais por causa do assédio, discriminação e até mesmo violência. Esses tipos de discriminação têm impacto sobre os níveis de escolaridade, uma vez que boa parte dessa parcela populacional evade ou é expulsa do ambiente escolar. Tais fatos tem consequências para outras questões, como renda, posições profissionais alcançadas, encarceramento, saúde e comportamento suicida, ao longo do ciclo de vida destes sujeitos (Grant, Mottet, Tanis, Herman, \& Keisling, 2011).

Uma das variáveis preditoras no modelo de regressão linear traçado, com o desfecho do escore de identidade social, foi o fato do participante estar ou não em tratamento hormonal. Ao tratar dessa questão, há uma fabricação do corpo feminino, mostrando que travestis ou mulheres trans realizam o tratamentos hormonais a fim de obter modificações corporais "como o desenvolvimento de seios, arredondamento e suavização dos joelhos, pernas, quadril e braços, diminuição da produção de pêlos no corpo", aplicação de silicone, além de "acuendar a neca", que se trata de esconder o genital masculino com auxílio de peça íntima feminina (Benedetti, 2005).

Neste sentido, a tarefa do profissional de saúde mental centra-se muito mais em incluir a preparação do cliente para viver a vida como pessoa trans. Profissionais da psicologia são encorajados a entender que a experiência de gênero de uma pessoa trans também pode ser moldada por outros aspectos importantes da identidade (por exemplo, idade, raça/etnia, orientação sexual) e que a importância dos diferentes aspectos da identidade pode evoluir à medida que a pessoa continua o desenvolvimento psicossocial em toda a vida, independentemente de completar uma transição social ou médica. Além disso, dado quão facilmente pressupostos ou estereótipos podem influenciar o tratamento, a prática baseada em evidências pode ser especialmente relevante para a prática psicológica. Esta deve ser utilizada para desenvolver competências para trabalhar com as pessoas trans e suas famílias, buscando conhecimentos básicos atualizados e compreensão da identidade e expressão de gênero, aprendendo a interagir de forma respeitosa e sem julgamento. (American Psychological Association, 2009; American Psychological Association, 2015; Bockting \& Coleman, 2007).

A variável idade também atuou como preditora para a identidade social na Regressão Linear que foi conduzida. Ao que cerca essa discussão, no estudo de Kennedy (2008), dados tirados de uma análise de um artefato online sugeriam que a idade média em que as pessoas trans se tornam conscientes de que são pessoas transgênero é de aproximadamente 8 anos de idade, e que mais de $80 \%$ das pessoas transgênero se tornam conscientes de que são trans antes de deixarem a escola primária. No entanto, foi encontrado no NTDS (Grant, Mottet, Tanis, Herman, \& Keisling, 2011) que as maiores diferenças acerca da identidade/expressão de gênero estavam entre faixas etárias. Para aqueles que já haviam transicionado, foi calculada a idade de transição (quando eles começaram a viver em tempo integral em um gênero diferente do seu sexo designado ao nascimento) e a maioria o fez entre 25 e 44 anos, uma vez que na idade adulta esses indivíduos sofreriam menor impacto de fatores (maioridade legal, contexto familiar, comunitário, estudantil ou profissional) que poderiam interferir na vivência da identidade social trans (Grant, et al., 2011).

Através das análises de variância observou-se que aqueles indivíduos que possuem maiores níveis de apoio familiar, obtêm uma maior satisfação com o suporte social. Em consonância a tais dados, Costa 
e Dell'Aglio (2009), afirmam que uma comunicação familiar efetiva suscita nos indivíduos maior destreza para lidar com situações adversas, uma vez que é efetivo na redução do impacto resultante a exposição a eventos da vida estressores.

Ademais, ainda no que diz respeito ao apoio familiar, também percebeu-se pelas análises ANOVA e pela correlação de Pearson, a influência desta variável para a autoestima. E concernente a esses dados, a pesquisa de Soares et al. (2011) relata que o processo de apoio depende da capacidade de pedir ajuda, da diferenciação de quem pede ajuda e de quem apoia, para se obter uma visão positiva da rede. Em concordância a esses dados, um estudo realizado com 245 jovens adultos LGBT nos Estados Unidos (Ryan, Russell, Huebner, Diaz, \& Sanchez, 2010) constatou que a aceitação da família na adolescência previu uma maior autoestima, apoio social e um melhor estado de saúde geral (incluindo taxas mais baixas de depressão, abuso de substâncias, ideação suicida e tentativas) na idade adulta jovem (Ryan et al., 2010).

A pesquisa conduzida por Snapp, Watson, Russel, Diaz e Ryan (2015) verificou que transexuais relataram níveis comparáveis de autoestima geral, apesar de relatarem significativamente menor satisfação com a sua situação de vida e baixa autoestima relacionado à pertença ao grupo LGBT (autoestima coletiva). Obter a sua identidade de gênero e a sua condição de ser trans reconhecida (coming out) para a família, amigos e colegas previu significativamente um melhor ajustamento, em particular à situação de vida e a estima em relação a grupo LGBT (Snapp, et al., 2015).

Ainda em relação à autoestima, uma possível explicação para a não correlação entre autoestima e a satisfação com o suporte social, pode ter se dado porque o instrumento de mensuração utilizado está atrelado a autoestima pessoal (Hutz, 2000; Rosenberg, 1965) e não a autoestima coletiva (Crocker \& Luhtanen, 1990), e da mesma forma poderia se explicar pelo fato da autoestima pessoal não influenciar na identidade social. Sugere-se também em futuras investigações o uso da medida de autoestima coletiva (Luhtanen \& Crocker, 1992), a fim de compreender a relação ou a influência desse construto sobre a identidade social.

Dessa forma, o apoio social é importante para pessoas trans na medida em que pode atenuar o estresse psicológico causado pela estigmatização (D'Augelli et al., 2001; Green, 2000) e a afiliação com comunidades LGBT pode fornecer um ambiente livre do estigma, bem como suporte para experiências negativas perpetradas por uma sociedade heterossexista, regida por um sistema ideológico que nega, denigre e estigmatiza qualquer forma não heterossexual de comportamento, identidade, relacionamento ou comunidade. Ademais, as comunidades LGBT também podem trazer um reflexo positivo ao indivíduo, permitindo-lhes comparar-se aos membros do grupo minoritário de pertença, ao invés dos membros da maioria heterossexual (Meyer, 2003; Monro, 2000).

\section{Considerações finais}

A experiência identitária trans traz à tona o questionamento acerca do que está pré-estabelecido para o entendimento da categoria gênero e sobre os padrões de normalidade e doença para as identidades de gênero e subjetividades, pois as histórias das(os) travestis, homens e mulheres trans e pessoas não binárias questionam a continuidade entre corpo, sexualidade e gênero (Bento, 2006). Em referência a esse aspecto, a luta pela despatologização da transexualidade e da travestilidade é uma das pautas que unifica teóricas(os) e ativistas de várias partes do mundo. Dessa forma, com base nas atuais mudanças da sociedade, ainda há pouca visibilidade no que diz respeito às questões ligadas à transgeneridade, muito ainda precisa ser feito e os cuidados para com esta parcela populacional precisam ser reafirmados.

Concernente a este aspecto, o presente estudo contribui para o aumento de conhecimento científico que possa direcionar profissionais das áreas sociais, de saúde e de educação, demistificando preconceito e estereótipos contra a diversidade de gênero, e auxiliar na construção de políticas públicas que possam atender a reais demandas desse grupo social. Neste sentido, buscou-se investigar a importância da rede de apoio social e do suporte oferecido pela mesma na autoestima e na identidade social, e consequentemente no sentimento de pertença de sujeitos trans à sociedade da qual fazem, integram, exercem seus deveres e, portanto, merecem representatividade e garantia dos direitos universais previstos por lei.

No que concerne a sugestões para estudos futuros, se faz necessário discutir e aprofundar mais questões como religiosidade, profissionais/laborais e de renda, de escolaridade, uma vez que estes indivíduos muitas vezes são excluídos do mercado de trabalho e evadem a escola por conta de discriminação e preconceito sofridos. Questões como a parentalidade trans ou cruzamento com outras variáveis que expressem outros tipos de preconceito como o racial ou análises entre pessoas binárias e não binária. 


\section{Referências}

Almeida, G. (2012). 'Homens Trans': novos matizes na aquarela das masculinidades? Revista Estudos Feministas (Florianópolis), 20(2), 256-266. https://doi.org/10.1590/S0104-026X2012000200012

Álvaro, J. \& Garrido, A. (2007). Psicologia Social-perspectivas psicológicas e sociológicas (M. C. Fernandes, Trad.) São Paulo: McGraw-Hill.

American Psychological Association. (2009). Report of the Task Force on Gender Identity and Gender Variance. Washington, DC: Author. http://www.apa.org/pi/lgbt/resources/policy/gender-identity-report.pdf

American Psychological Association. (2015). Guidelines for Psychological Practice With Transgender and Gender Nonconforming People. American Psychologist, 70(9), 832-864. https://doi.org/10.1037/a0039906

Barišic, J., Milosavljevic, M., Duišin, D, Batinic, B. Vujovic, S., \& Milovanovic, S. (2014). Assessment of Self-Perception of Transsexual Persons: Pilot Study of 15 Patients. The Scientific World Journal, Hindawi Publishing Corporation, 1-5. https://doi.org/10.1155/2014/281326

Benedetti, M. R. (2005). Toda Feita: O corpo e o gênero das travestis. Rio de Janeiro: Garamond.

Bento, B. (2006). A reinvenção do corpo: sexualidade e gênero na experiência transexual. Rio de Janeiro: Garamond.

Bento, B. (2009). A diferença que faz a diferença: corpo e subjetividade na transexualidade. Bagoas, 4(1), 95-112.

Brasil. Ministério da Saúde (2009). Portaria no 1.820, 13 de agosto de 2009. Dispões sobre os direitos e deveres dos usuários da saúde. Brasília: Diário Oficial da União.

Bockting, W., Benner, A., \& Coleman, E. (2009). Gay and bissexual identity development among female-to-male transsexuals in North America: emergence of a transgender sexuality. Archives of Sexual Behavior, 38(5), 688-701. https://doi.org/10.1155/2014/281326

Costa, L. G. \& Dell'Aglio, D. D. (2009). A rede de apoio social de jovens em situação de vulnerabilidade social. In R. M. C. Libório \& S. H. Koller (Eds.). Adolescência e juventude: risco e proteção na realidade brasileira (pp. 219-263). São Paulo: Casa do Psicólogo.

Crocker, J. \& Luhtanen, R. (1990). Collective self-esteem and ingroup bias. Journal of Personality and Social Psychology, 58(1), 60-67. https://doi.org/10.1037/0022-3514.58.1.60

Deschamps, J. (2009). A identidade em Psicologia Social: dos processos identitários às representações sociais (M. Lucia, Trad.). Petrópolis, Rio de Janeiro: Vozes.

Grant, J. M., Mottet, L. A., Tanis, J. D. J., Herman, J. H. J. L., \& Keisling, M. (2011). Injustice at Every Turn: A Report of the National Transgender Discrimination Survey. Washington: National Center for Transgender Equality and National Gay and Lesbian Task Force.

Green, J. (2000). Introduction to transgender issues. In P. Currah, \& S. Minter, S. Transgender equality: A handbook for activists and policymakers (pp. 1-12). New York: The National Gay and Lesbian Task Force Policy Institute.

Grossman, A. H., D’augelli, A. R. \& Frank, J. A. (2011). Aspects of Psychological Resilience among Transgender Youth. Journal of LGBT Youth, 8(2), 103-115. https://doi.org/10.1080/19361653.2011.541347

Hutz, C. (2000). Adaptação brasileira da Escala de Autoestima de Rosenberg. Curso de Pós-Graduação em Psicologia, Universidade Federal do Rio Grande do Sul, Porto Alegre. Mimeo.

Kennedy, N. (2008). Transgendered Children in Schools: a critical review of homophobic bullying: safe to learn, embedding anti-bullying work in schools. Forum, 50(3), 383-396. https://doi.org/10.2304/forum.2008.50.3.383

Koller, S. H., Cerqueira-Santos, E., Morais, N. A., \& Ribeiro, J. (2005). Juventude brasileira. Relatório técnico para o Banco Mundial. Washington, DC: World Bank.

Koller, S. H., Cerqueira-Santos, E., Morais, N. A., \& Ribeiro, J. (2005). Juventude brasileira: fatores de risco e de proteção. Relatório de pesquisa - World Bank. Porto Alegre, RS.

Luhtanen, R. \& Crocker, J. (1992). A collective self-esteem scale: Self-evaluation of one's social identity. Personality \& Social Psychology Bulletin, 18(3), 302-318. https://doi.org/10.1177/0146167292183006

Mayhew, M. J., Gardner, J., \& Achkanasy, N. M. (2010). Measuring individuals' need for identification: Scale development and validation. Personality and Individual Differences, 49(50), 356-361. https://doi.org/10.1016/j.paid.2010.03.031

Meyer, I. H. (2003). Bias, social stress, and mental health in lesbian, gay, and bisexual populations: Conceptual issues and research evidence. Psychological Bulletin, 129(5), 674-697. https://doi.org/10.1037/0033-2909.129.5.674

Monro, S. (2000). Theorizing transgender diversity: Towards a social model of health. Sexual and Relationship Therapy, 15(1), 33-45. https://doi.org/10.1080/14681990050001547

Nelson, T. (2002). Chapter 2: Origin and Maintenance of Stereotypes and Prejudice. In: The psychology of prejudice. Boston, MA: Modern Graphics, Inc., 46-48.

Paludo, S. \& Koller, S. H. (2004). Inserção ecológica no espaço da rua. In S. H. Koller (Ed.), Ecologia do desenvolvimento humano: pesquisa e intervenção no Brasil. São Paulo: Casa do Psicólogo, 123-154.

Paris-Ribeiro, J. L. (1999). Escala de Satisfação com o Suporte Social. Análise Psicológica, 28(3), 547-558.

Pinto, M. J. C. \& Bruns, M. A. T. (2006). A transexualidade no contexto hospitalar. In M. C. O. S. Miyazaki, N. A. M. Domingos, \& N. I. Valerio (Orgs.). Psicologia da saúde: Pesquisa e prática (pp. 401-422). São José do Rio Preto, SP: THS/Arantes Editora. 
Próchno, C. C. S. C. \& Rocha, R. M. G. (2011). O jogo do nome nas subjetividades travestis. Psicologia \& Sociedade, 23(2), 254-261. https://doi.org/10.1590/S0102-71822011000200006

Ribeiro, J. L. P. (1999). Escala de Satisfação com o Suporte Social. Analise Psicológica, 3(XVIII), 547-558.

Rosenberg, M., Schooler, C., Schoenbach, C., \& Rosenberg, F. (1995). Global self-esteem and specific self-esteem: different concepts, different outcomes," American Sociological Review, 60(1), 141-156. https://doi.org/10.2307/2096350

Ryan, C., Russell, S. T., Huebner, D., Diaz, R., \& Sanchez, J. (2010). Family acceptance in adolescence and the health of LGBT young adults. Journal of Child and Adolescent Psychiatric Nursing, 23(4), 205-213. https://doi.org/10.1111/ j.1744-6171.2010.00246.x

Sánchez, F. J. \& Vilain, E. (2009). Collective Self-Esteem as a Coping Resource for Male-to-Female Transsexuals, Journal of Counseling Psychology, American Psychological Association, 56(1), 202-209. https://doi.org/10.1037/ a0014573

Silva, B. B. \& Cerqueira-Santos, E. (2014). Apoio e Suporte social na Identidade social de travestis, transexuais e transgêneros. Revista da Sociedade de Psicoterapias Analíticas Grupais do Estado de São Paulo - SPAGESP (São Paulo), 15(2), 27-44. Acesso em: 11 jul. 15. Disponível em: http://pepsic.bvsalud.org/scielo.php?pid=S167729702014000200004\&script=sci arttext

Snapp, S. D., Watson, R. J., Russel, S., Diaz, R. M., \& Ryan, C. (2015). Social Support Networks for LGBT Young Adults: Low Cost Strategies for Positive Adjustment. Family Relations: Interdisciplinary Journal of Applied Family Studies, 64(3), 420-430. https://doi.org/10.1111/fare.12124

Soares, M., Feijó, M. R., Valério, N. I., Siquieri, C. L. S. M., \& Pinto, M. J. C. (2011). O apoio da rede social a transexuais femininas. Paidéia, 21(48), 83-92. https://doi.org/10.1590/S0103-863X2011000100010

Sousa, L. \& Suda, J. R. (2006). Identidade Social em Movimento: A comunidade japonesa na grande Vitória. Psicologia \& Sociedade (Porto Alegre), 18(2), 72-80. https://doi.org/10.1590/S0102-71822006000200010

Stotzer, R. L. (2011). Family Cohesion among Hawai'i’s Mahuwahine. Journal of GLBT Family Studies, 7(5), 424-435. https://doi.org/10.1080/1550428X.2011.623935

Tajfel, H. (1981). Human Groups and Social Categories. Cambridge: Cambridge University Press.

Winck, G. E. \& Petersen, C. M. S. (2005). O transexual masculino: considerações sobre rede de apoio social e coping na mudança do sexo anatômico. Revista Ártemis, 2, 26-39.

Dados dos autores:

Bruno de Brito Silva - Doutorando, Universidade Federal do Rio Grande do Sul.

Elder Cerqueira-Santos - Pós-Doutorado, Universidade Federal de Sergipe.

\section{Endereço para correspondência:}

Bruno de Brito Silva

Rua Construtora Cunha, 69, Condomínio Vales dos Lagos - Vitória, 703

49027-340 Aracaju, SE, Brasil

<bruno.bbs8@gmail.com>

Recebido em: 02.03.2018

Aceito em: 13.06.2018 